\title{
Tunnel-Coupled Quantum Dots: Atomistic Theory of Quantum Dot Molecules and Arrays
}

\author{
Garnett W. Bryant ${ }^{1}$, Javier Aizpurua ${ }^{1}$, W. Jaskolski ${ }^{2}$ and Michal Zielinski ${ }^{2}$ \\ ${ }^{1}$ Atomic Physics Division, National Institute of Standards and Technology, Gaithersburg, MD \\ 20899-8423 \\ ${ }^{2}$ Instytut Fizyki, UMK, Grudziadzka 5, 87-100 Torun, Poland
}

\begin{abstract}
An understanding of how dots couple in quantum dot molecules and arrays is needed so that the possibilities for tailored nanooptics in these systems can be explored. The properties of tunnel-coupled dots will be determined by how the dots couple through atomic-scale junctions. We present an atomistic empirical tight-binding theory of coupled, CdS nanocrystal artificialmolecules, vertically and laterally coupled InAs/GaAs self-assembled dots, and arrays of InAs/GaAs self-assembled dots. Electron states follow the artificial molecule analogy. The coupling of hole states is much more complex. There are significant departures from the artificial molecule analogy because the interdot hole coupling is determined by the hole envelope functions, as for the electron states, and by the hole atomic state near interdot interfaces.
\end{abstract}

\section{INTRODUCTION}

Quantum dots and nanocrystals have been studied intensely due to their enticing possibilities as artificial atoms with enhanced optical properties. It is now possible to envision using these artificial atoms as the building blocks for novel artificial solids or as the device elements in complex nanoarchitectures. Artificial solids could be constructed from dots without the limitations on geometry and composition normally imposed by chemical bonding in natural solids. Nanoarchitectures of dots provide the possibility of pushing classical computing almost to the atomic regime, providing coherent quantum structures needed for quantum computing, and bridging the interface to biological systems with bio/nanohybrids. Construction of artificial solids, nanoarchitectures and bio/nanohybrids is a challenging, ongoing problem. Ensembles of quantum dots and nanocrystals ordered in one, two, and three dimensions are being studied [1-5]. An understanding of how dots should couple and function in these structures is needed so that the possibilities for tailored nanooptics in these systems can be explored. The properties of tunnel-coupled quantum dots will be determined by how the dots couple through atomic-scale tunnel junctions. Here we present an atomistic empirical tight-binding theory of coupled, $\mathrm{CdS}$ nanocrystal artificial-molecules, vertically and laterally coupled InAs/GaAs self-assembled dots, and one-dimensional and two-dimensional arrays of InAs/GaAs self-assembled dots.

\section{THEORY}

Electron and hole states in these nanoparticle nanosystems are calculated by use of the 
empirical tight-binding (ETB) method [6-12]. The ETB approach is an atomistic approach well suited for calculating the electronic states of nanosystems with atomic-scale interfaces and variations in composition and shape. Our ETB theory can be used to model single dots or coupled dot systems. In this paper, we focus on dot-molecules made by coupling two dots and dot arrays formed by coupling one-dimensional arrays of vertically-stacked, identical selfassembled dots or two-dimensional arrays of laterally coupled, identical self-assembled dots. We assume that atoms in the individual dots occupy the sites of a regular zinc-blende lattice. We can model spherical, hemispherical, tetrahedral or pyramidal nanoparticles. To study the effects of coupling nanocrystals together, we assume that the nanocrystals are connected epitaxially at the interface between the dots with the same lattice in each dot. In practice, this need not be the case. However, this assumption greatly simplifies the model for these initial calculations. To study coupled self-assembled dots, we model the dots, wetting layers, and a box made of the capping material. The box defines the supercell for the array calculations.

In our ETB model, each atom is described by its outer valence s orbital, the 3 outer $p$ orbitals and a fictitious excited $\mathrm{s}^{*}$ orbital, included to mimic the effects of higher lying states [13]. The empirical Hamiltonians are determined by adjusting the matrix elements to reproduce band gaps and effective masses of the bulk band structures. We include on-site and nearestneighbor coupling between orbitals. Spin-orbit coupling has also been included in the theory but will not be considered for the results presented here. This simplification allows us to focus on how the spatial distribution and atomic orbital of the dot state determines the interdot coupling and hybridization.

The electron and hole eigenstates close to the band edges are found by diagonalizing the Hamiltonians with an iterative eigenvalue solver. The nanosystems have on the order of 500025,000 atoms. Typically, we find the several hundred states closest to the band edge. We explicitly exclude the effects of surface states in our calculations by passivating the surface dangling bonds. The passivation is modeled by shifting the energy of the dangling bonds above the conduction band so that the dangling bonds do not modify states near the band gap. No lattice relaxation is included. This is a significant approximation for the self-assembled dots. We make the approximation here so that we can focus on the effects of interdot coupling and hybridization. We model dot molecules made from CdS nanocrystals with the CdS ETB parameters taken from Lippens and Lannoo [6]. Calculations for self-assembled dots are done for InAs/GaAs dots with the InAs and GaAs ETB parameters taken from Vogl et al. [13].

Optical spectra are calculated by evaluating the dipole matrix elements for electron and hole eigenstates found in the ETB calculations [7,8]. In the ETB approach, dipole matrix elements are not needed to define the Hamiltonian. We find on-site dipole matrix elements by use of calculated atomic dipole matrix elements [14]. Dipole matrix elements between bonding orbitals on nearest neighbor sites are chosen by reasonable estimates. The qualitative structure in the calculated spectra is insensitive to variations in the dipole matrix elements for bond orbitals for choices of these matrix elements less than the bond length. This shows that allowed transitions are determined by symmetry considerations.

\section{RESULTS}

Electron states of coupled dots follow closely the analogy that one would expect of coupled artificial atoms as artificial molecules. Figure 1 shows how the electron levels change as the center-to-center separation between two coupled spherical CdS nanocrystals is varied. 


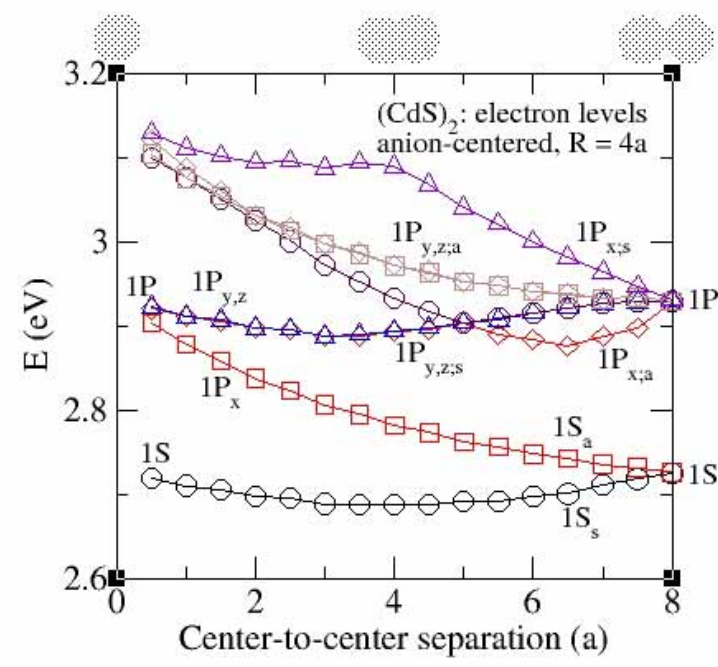

Figure 1. Electron levels for two coupled spherical CdS nanocrystals (radius $\mathrm{R}=4 \mathrm{a}$, where a is the CdS lattice constant). Dependence on center-to-center separation is shown. Dots separated by $8 \mathrm{a}$ are touching at one atom. Zero separation corresponds to two dots completely overlapped. State symmetries are indicated: $a$ and $s$ indicate antisymmetric and symmetric pairing, a subscript $x, y$, or $z$ indicates the symmetry of the $\mathrm{P}$ envelope. The dot-molecule axis is along $\mathrm{x}$.

Symmetric/antisymmetric (bonding/antibonding) pairs are formed in the coupled dots. The coupling is strongest between states with high density at interdot interfaces. In coupled spherical nanocrystals, $1 \mathrm{~S}$ electron intradot states can couple strongly. 1P states that lie along the molecular axis couple more strongly to form $\sigma$-like bonds while 1P states perpendicular to the molecular axis couple more weakly to form $\pi$-like bonds. Symmetric coupling gives the lower energy state if the two intradot states that are coupled have the same phase at the common interface. Antisymmetric coupling gives the lower state if the two intradot states have opposite phase at the interface. Similar effects are seen for electron states in coupled self-assembled dots.

The coupling of hole states is much more complex. There are significant departures from the artificial molecule analogy because the interdot hole coupling is determined by the hole envelope functions, as in the case of the electron states, and by the hole atomic state, especially at the interdot interface. As an example, the hole levels for two coupled CdS nanocrystals are shown in figure 2 as a function of dot separation. Some hole states couple by forming symmetric/antisymmetric pairs. Other hole states couple by mixing through additional intermediate states. This leads to the formation of two symmetric states that are strongly split by

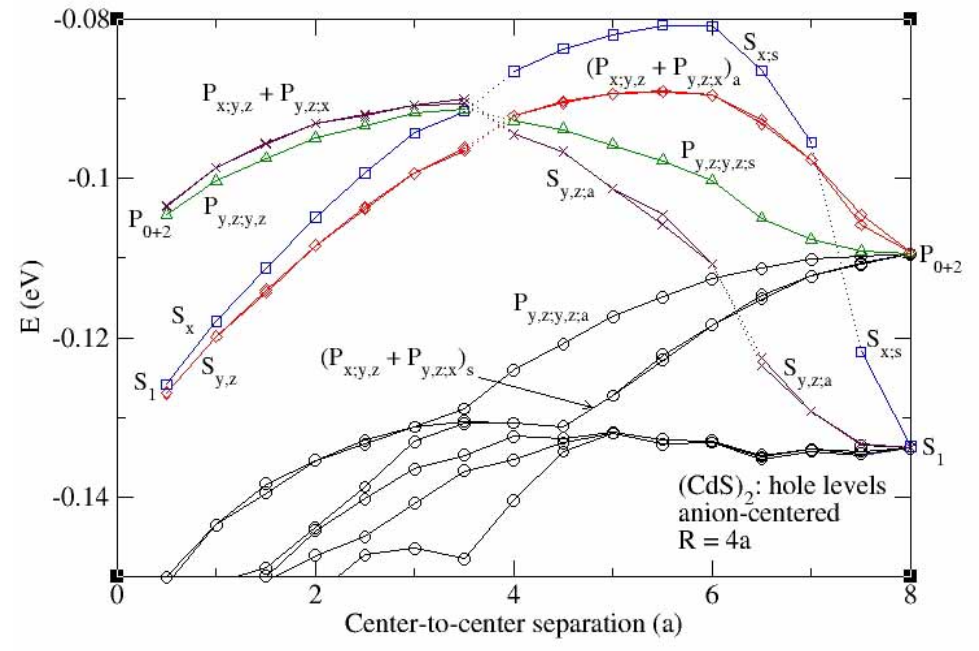

Figure 2. Dependence of hole levels for two coupled spherical CdS nanocrystals $(\mathrm{R}=4 \mathrm{a})$ on center-tocenter separation. State symmetries are shown: $a$ and $s$ for antisymmetric and symmetric pairing, a single subscript $x, y$, or $z$ indicates the dominant $\mathrm{p}$ atomic orbitals on the anions, a subscript of the form $x ; y$ indicates first the symmetry of the $\mathrm{P}$ envelope and second the dominant $\mathrm{p}$ atomic orbitals. The dot-molecule axis is along $\mathrm{x}$. 
coupling and an antisymmetric state which is insensitive to the coupling. For some hole states, the role of symmetric and antisymmetric pairing is reversed because of the atomic character of the hole state near the interface. For example, the lowest hole state for the isolated dot has a $\mathrm{P}$ envelope. When the dots slightly overlap, this splits into a symmetric/antisymmetric pair with the antisymmetric state giving the lower energy. For holes, the total phase of the state, that is due to both the envelope phase and the phase of the $\mathrm{p}$ atomic orbitals, near the interface, determines whether the symmetric or antisymmetric state has the lower energy. The lowest S state of the isolated dot couples through an intermediate interface state and experiences a much larger splitting than the lowest $\mathrm{P}$ state. As a consequence, the $\mathrm{S}$ state becomes the hole ground state for intermediate dot center-to-center separation.

Intradot coupling has important effects on the nanooptics of these nanosystems: level reordering, changes in state symmetry, conversion of dark states to bright states and vice versa, and tailored polarization dependence. The electron and hole level structure and allowed transitions in the coupled CdS double-dot for separations D = a and 6 a are shown in figure 3. Level symmetries and degeneracies are indicated. When the two dots are nearly completely overlapping (i.e. almost the limit of a single dot) the only active transitions are those which are dipole-allowed by the intradot spatial symmetry of the electron and hole states. In this case, the lowest-energy transition between electron and hole states is dipole forbidden. For the double-dot molecule with $\mathrm{D}=6 \mathrm{a}$, dipole transitions allowed by the intradot symmetry still occur. However, in this case, the order of the lowest hole levels is different, as shown in figure 2, and the lowestenergy transition is dipole allowed. At the same time, new transitions are allowed. These overlap-allowed transitions arise when a significant portion of the electron and hole states reside in the region where the dots overlap. In this case, the symmetry of the electron and hole states in the overlap region determines the dipole-allowed transitions.

The complex evolution of hole states in coupled dots can be used to understand the level shifting and broadening observed during the formation of quantum rods [15] and stacked selfassembled quantum dots [1]. However, there are interesting differences between coupled dots and coupled self-assembled dots. These differences arise, in part, because nanocrystals must touch or overlap to couple via tunneling while self-assembled dots can couple via tunneling through the capping material without touching. For example, the lowest hole state in a selfassembled dot is heavy-hole like. However, the confinement by the dot mixes in a small lighthole component as well. Just outside the self-assembled dot, the heavy-hole component remains

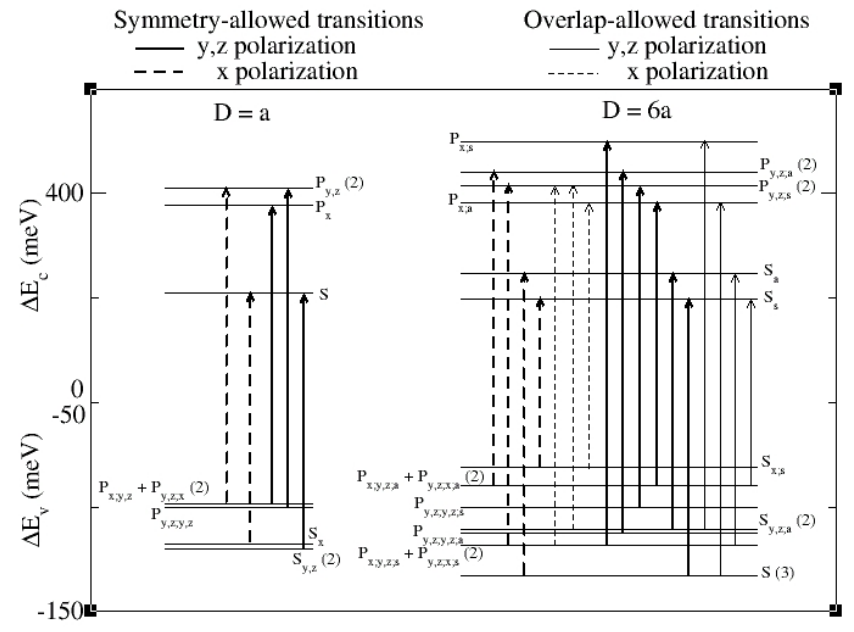

Figure 3. Level structure and allowed transitions in the CdS double-dot $(\mathrm{R}=$ 4a) for separation $\mathrm{D}=\mathrm{a}$ and $6 \mathrm{a}$. State symmetries are shown: $a$ and $s$ for antisymmetric and symmetric pairing, a single subscript $x, y$, or $z$ indicates the dominant $\mathrm{p}$ atomic orbitals on the anions, a subscript of the form $x$; $y$ indicates indicates first the symmetry of the $\mathrm{P}$ envelope and second the dominant $p$ atomic orbitals, the state degeneracy is given. The dot-molecule axis is along $\mathrm{x}$. 
dominant. However, the heavy-hole component decays more quickly than the light-hole component in the barrier. Far from the dot, only the light-hole component remains. For widely separated vertical-stacked self-assembled dots, the coupling is through the light-hole component. This coupling favors antisymmetric coupling. For closely spaced, vertical-stacked self-assembled dots, the coupling is through the heavy-hole component and symmetric coupling is favored. The lowest electron-hole transitions in the single self-assembled InAs/GaAs dot are dipole-allowed. For closely spaced, self-assembled dots, the lowest electron-hole transitions remain allowed because both electron and hole states in the coupled structure are symmetric states. For widely spaced, self-assembled dots, the lowest electron-hole transitions are forbidden because the hole states are antisymmetric. This conversion between bright and dark transitions in vertically stacked self-assembled dots comes about because of a reversal in the strength of symmetric/antisymmetric coupling that happens without reordering the intradot symmetry of the levels. The conversions that we have discussed in this paper for coupled nanocrystals come about because the coupling reorders levels that have different intradot symmetry.

The dependence of the strength of symmetric/antisymmetric coupling on the separation between vertical stacked self-assembled dots affects the band structure of one-dimensional arrays of vertically stacked dots. In an array, band states are made by coupling the states from the individual dots. Symmetric interdot coupling gives the zone-center states, while antisymmetric interdot coupling gives the zone-edge states. For an array of closely spaced, vertically stacked dots, symmetric coupling is favored and the lowest hole bands have normal dispersion. For widely spaced dots, antisymmetric coupling is favored and the lowest hole bands have negative dispersion.

\section{CONCLUSIONS}

An understanding of how dots couple in quantum dot molecules and arrays is needed so that the possibilities for tailored nanooptics in these systems can be explored. We present an atomistic empirical tight-binding theory of coupled, $\mathrm{CdS}$ nanocrystal artificial-molecules, vertically and laterally coupled InAs/GaAs self-assembled dots, and arrays of InAs/GaAs selfassembled dots. Electron states follow the artificial molecule analogy. The coupling of hole states is much more complex. There are significant departures from the artificial molecule analogy because the interdot hole coupling is determined by the hole envelope functions, as for the electron states, and by the hole atomic state near interdot interfaces. The coupling can have important effects on the nanooptics of these nanosystems: level reordering, changes in state symmetry, conversion of dark states to bright states and vice versa, and tailored polarization dependence. The complex evolution of hole states in coupled dots can be used to understand the level shifting and broadening observed during the formation of quantum rods and stacked selfassembled quantum dots and can produce hole states with negative dispersion in self-assembled dot arrays.

\section{REFERENCES}

1. G. S. Solomon, J. A. Tressa, A. F. Marshall, and J. S. Harris, Jr., Phys. Rev. Lett. 76, 952 (1996).

2. B. Grandidier, Y. M. Niquet, B. Legrand, J. P. Nys, C. Priester, D. Stievenard, J. M. Gerard, 
and V. Thierry-Mieg, Phys. Rev. Lett. 85, 1068 (2000).

3. R. L. Williams, G. C. Aers, P. J. Poole, J. Lefebvre, D. Chithrani, and B. Lamontagne, J. Crystal Growth, 223, 321 (2001).

4. C. R. Kagan, C. B. Murray, M. Nirmal, and M. G. Bawendi, Phys. Rev. Lett. 76, 1517 (1996).

5. M. V. Artemyev, A. I. Bibik, L. I. Gurinovich, S. V. Gaponenko, and U. Woggon, Phys. Rev. B 60, 1504 (1999).

6. P. E. Lippens and M. Lannoo, Phys. Rev. B 39, 10935 (1989).

7. K. Leung and K. B. Whaley, Phys. Rev. B 56, 7455 (1997).

8. K. Leung, S. Pokrant, and K. B. Whaley, Phys. Rev. B 5, 12291 (1998).

9. G. W. Bryant and W. Jaskolski, Phys. St. Sol. B 224, 751 (2001).

10. G. W. Bryant and W. Jaskolski, Physica E 11, 72 (2001).

11. G. W. Bryant and W. Jaskolski, Physica E 13, 293 (2002).

12. G. W. Bryant, Physica B 314, 15 (2002).

13. P. Vogl, H. P. Hjalmarson, and J. D. Dow, J. Phys. Chem. Solids 44, 365 (1983).

14. S. Frage and J. Muszynska, Atoms in External Fields, (Elsevier, New York 1981).

15. J. Hu, L.S. Li, W. Yang, L. Manna, L.-W. Wang, and A. P. Alivisatos, Science 292, 2060 (2001). 